\title{
Investigation of the Interaction between Mucins and -Lactoglobulin under Tribological Stress
}

\author{
Celebioglu, Hilal Yilmaz; Guðjónsdóttir, María; Chronakis, loannis S.; Lee, Seunghwan
}

Published in:

Food Hydrocolloids

Link to article, DOI:

10.1016/j.foodhyd.2015.09.013

Publication date:

2016

Document Version

Peer reviewed version

Link back to DTU Orbit

Citation (APA):

Celebioglu, H. Y., Guðjónsdóttir, M., Chronakis, I. S., \& Lee, S. (2016). Investigation of the Interaction between Mucins and -Lactoglobulin under Tribological Stress. Food Hydrocolloids, 54(Part A), 57-65.

https://doi.org/10.1016/j.foodhyd.2015.09.013

\section{General rights}

Copyright and moral rights for the publications made accessible in the public portal are retained by the authors and/or other copyright owners and it is a condition of accessing publications that users recognise and abide by the legal requirements associated with these rights.

- Users may download and print one copy of any publication from the public portal for the purpose of private study or research.

- You may not further distribute the material or use it for any profit-making activity or commercial gain

- You may freely distribute the URL identifying the publication in the public portal 
1

\title{
Investigation of the Interaction between Mucins and $\beta$-Lactoglobulin under
}

\author{
Tribological Stress
}

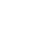

Hilal Y. Çelebioğlu, ${ }^{1}$ María Gudjónsdóttir, ${ }^{1 \dagger}$ Ioannis S. Chronakis, ${ }^{1}$ and Seunghwan Lee ${ }^{2 *}$

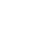

${ }^{1}$ Nano-BioScience Research Group, DTU-Food, Technical University of Denmark, Søltofts plads, building 227, 2800 Kgs. Lyngby, Denmark.

${ }^{2}$ Department of Mechanical Engineering, Technical University of Denmark, DK-2800 Kgs. Lyngby, 10

\section{Denmark}

Keywords: tribology, beta-lactoglobulin, bovine submaxillary mucin, porcine gastric mucin, $\mathrm{pH}$

*Corresponding author: Tel: +4545252193, e-mail: $\underline{\text { seele@mek.dtu.dk }}$

$\dagger$ Permanent address: University of Iceland, Faculty of Food Science and Nutrition, Vínlandsleið 14, 113 Reykjavík, Iceland

\begin{abstract}
The interaction characteristics between mucins and beta-lactoglobulin (BLG) under tribological stress were investigated by comparing the lubricity of mixed solutions of mucin-BLG with that of neat protein solutions at compliant hydrophobic interfaces. Surface adsorption properties of the proteins as characterized by bicinchoninic acid (BCA) assay revealed that both bovine submaxillary mucin (BSM) and porcine gastric mucin (PGM) showed distinctly higher adsorbed masses compared to BLG onto polydimethylsiloxane (PDMS) or polystyrene (PS) surfaces. The adsorbed
\end{abstract}


masses of the mixed protein solutions, namely BLG-BSM and BLG-PGM, reduced significantly, and BLG appeared to dominate the surface adsorption event, presumably due to the reduced concentration of mucins and the Vroman effect. While pin-on-disk tribometry and mini-traction machine (MTM) were employed to provide the tribological contacts with varying contact pressure, speed range, and slide/roll ratio, the dominant lubrication mechanism of the protein solutions was boundary lubrication. BLG-BSM mixture showed the highest level of degradation in the lubricity of $\mathrm{BSM}$ at $\mathrm{pH} 5$, although BLG-saliva interaction is known to degrade the lubricity most rapidly at more acidic $\mathrm{pH}$, such as at $\mathrm{pH} 3.5$. More importantly, $\mathrm{pH}$ dependent lubricating properties of BLGBSM mixed solutions appeared to be determined by competitive adsorption of the two proteins onto the substrates, which suggests that they do not form as strong aggregates as BLG-saliva, especially under tribological stress.

\section{Introduction}

There has been growing interest in understanding food oral processing and digestion by applying various techniques to achieve desired designing of food and pharmaceuticals with new ingredients and interfacial structures (Lundin, Golding, \& Wooster, 2008; McClements, Decker, \& Park, 2009;

Singh, Ye, \& Horne, 2009; Singh \& Ye, 2013). A few studies have investigated food oral processing by focusing on the interaction of food emulsions with saliva in the oral environment (Vingerhoeds et al. 2005; van Aken, Vingerhoeds, \& de Hoog, 2007; Silletti et al. 2007; Sarkar, Goh, \& Singh, 2009). These studies have shown that either electrostatic interaction or hydrophobic forces causes emulsion flocculation, aggregation, or aroma releasing, which are related to sensory perception. Due to complexity of both food and saliva, the details of food-saliva interactions still require further explanations. In particular, little information is available in literature on the molecular-level interaction between constituents of food-saliva systems. 
Recently, tribology has emerged as a new instrumental approach to investigate oral processing of food emulsions in simulated oral environment (Meyer et al. 2011; Vardhanabhuti et al. 2011; Chojnicka-Paszun, de Jongh, \& de Kruif, 2012; Chen \& Stokes, 2012; van Aken, 2013; Selway \& Stokes, 2013; Prakash, Tan, \& Chen, 2013; Chen, Liu, \& Prakash, 2014; Joyner Melito, Pernell, \& Daubert, 2014). In turn, this is often correlated with food's sensory perception (Meyer et al. 2011; Vardhanabhuti et al. 2011; Chojnicka-Paszun, de Jongh, \& de Kruif, 2012; Selway \& Stokes, 2013; Prakash, Tan, \& Chen, 2013). Tribology is particularly useful for understanding the behavior of thin films formed between two opposing surfaces where rheological and structural/mechanical properties of food may no longer explain their behavior sufficiently.

In the present study, we attempted to apply tribological techniques to investigate the interaction of $\beta$-lactoglobulin (BLG) with mucins under tribological stress and how it affects their lubricating properties. BLG is the major whey protein constituting $>50 \%$ of the total whey proteins in bovine milk (Zúñiga, Tolkach, Kulozik, \& Aguilera, 2010). BLG contains many charged groups, therefore, its structure and properties are highly pH-dependent (Fang \& Dalgleish, 1997). Mucins are a family of large, extracellular glycoproteins (Bansil, Stanley, \& LaMont, 1995; Svensson, \& Arnebrant, 2010) and are known to be chiefly responsible for the slipperiness of saliva (Tabak, Kevine, Mandel, \& Ellison, 1982; Berg, Lindh \& Arnenrant, 2004). Apart from its functions in biological systems, previous studies have shown facile adsorption and effective lubrication on various engineering materials too (Lee, Müller, Rezwan, \& Spencer, 2005; Yakubov, McColl, Bongaerts \& Ramsden, 2009; Nikogeorgos, Madsen, \& Lee, 2014). The importance of understanding the interaction characteristics between BLG and mucins is related to an ongoing discussion on the origin of astringency. One of the most prevailing models is that astringents interact with saliva to form aggregates to deplete the lubricant (saliva) from the tribological contacts in the mouth (Rossetti, Bongaerts, Wantling, Stokes, \& Williamson, 2009; Vardhanabhuti et al., 
2011). Recent applications of tribological techniques allowed for quantitative characterization of the lubricating properties of the fluids involving saliva and BLG or other astringents. For example, Vardhanabhuti et al. (2011) showed that addition of BLG into a soft tribological interface increased the interfacial friction forces, yet at varying rates depending on $\mathrm{pH}$. Aggregation of macromolecules (BLG) with hydrogel (saliva) is, however, a complex process influenced by a number of parameters. For example, among many types of proteins in saliva, which one(s) are involved in the aggregation with BLG is not clear. Thus, it would be important and meaningful to investigate the molecular-level interaction to deepen the understanding the interaction of saliva with whole food. Moreover, despite relatively more active studies addressing the interactions of mucins with polysaccharides (Qaqish, \& Amiji, 1999; Menchicchi et al., 2014; Menchicchi et al., 2015), the studies for the interaction of mucins with food proteins are much more limited.

We have chosen two types of mucins, namely, bovine submaxillary mucin (BSM) and porcine gastric mucin (PGM), purchased from a commercial manufacturer (Sigma-Aldrich). The fact that both mucins are highly relevant to food digestion process, yet to different organs, is the first reason for comparing them. Additionally, in parallel with common structural features of the two mucins (Bansil, Stanley, \& LaMont, 1995; Sandberg, Blom, \& Caldwell, 2009), reported differences in their biophysical properties, especially the lubricating properties (Lee, Müller, Rezwan, \& Spencer, 2005; Nikogeorgos, Madsen, \& Lee, 2014), may lead to different interaction with BLG and alteration in the lubricating properties.

\section{Materials and methods}

\subsection{Sample preparation}

BLG from bovine milk, BSM (Type I-S), and PGM (Type III) were purchased from Sigma-Aldrich (Brøndby, Denmark), and were used as received. Protein solutions with the concentration of 1 
$\mathrm{mg} / \mathrm{mL}$ were prepared by dissolving in $10 \mathrm{mM}$ phosphate buffered saline (PBS) solutions and were used throughout the study. The $\mathrm{pH}$ values of the buffer solutions were adjusted to $7.4,5$, and 3 by addition of $\mathrm{HCl}$ or $\mathrm{NaOH}$. For the mixture of $\mathrm{BLG}$ and mucins, the two protein solutions were mixed directly at the ratio of $1: 1(\mathrm{v}: \mathrm{v})$ and the total protein concentrations were remained at either 1 $\mathrm{mg} / \mathrm{mL}$. Due to relatively weaker lubricating capabilities of PGM at $1 \mathrm{mg} / \mathrm{mL}$ (Lee, Müller, Rezwan, \& Spencer, 2005), PGM, BLG, and PGM-BLG mixed solutions were studied at $10 \mathrm{mg} / \mathrm{mL}$ too.

\subsection{Zeta potential measurements}

The zeta potential of the protein solutions was characterized with a laser $(633 \mathrm{~nm})$ Doppler electrophoresis instrument (LDE; Zetasizer Nano ZS, Malvern, UK). Disposable cuvettes (model DTS 1070) were used. At least five measurements were performed for each protein solution to acquire statistically valid data.

\subsection{Bicinchoninic acid (BCA) assay}

Surface adsorption properties of the proteins onto hydrophobic substrates were characterized by means of BCA protein qualification assay. This technique is based on the reduction of $\mathrm{Cu}^{2+}$ to $\mathrm{Cu}^{+}$ in the presence of peptide bonds, and subsequent complex formation with BCA to form a purplecolored end product (Smith et al. 1985). The assay has been established to quantify proteins in bulk solution, and recently, it was further customized to estimate the protein amount adsorbed on surfaces (Sandberg, Mellina, Gelius, \& Caldwell, 2009; Pakkanen, Madsen, \& Lee, 2015). An important assumption in this modified approach is that surface adsorbed proteins (peptide bonds) have the same reduction reactivity of converting $\mathrm{Cu}^{2+}$ to $\mathrm{Cu}^{+}$with those in bulk solution. Thus, the light absorbance by $\mathrm{Cu}^{+}-\mathrm{BCA}$ complex is proportional to the amount of the proteins even if the 
proteins are surface-localized. Detailed procedures to acquire standard curves and the areal mass of the sample proteins are provided in the Supplementary Information (Figure S1.) In this study, standard curves were prepared for not only bovine serum albumin (BSA) as a general test protein, but also for all the proteins or protein mixtures at each condition, including concentration, $\mathrm{pH}$, and the choice of microtiter materials (see below). The absorbance of each protein sample was measured at $540 \mathrm{~nm}$ with an absorbance microplate reader (BioTek, ELx800 model). The measurements were repeated three times for each sample for statistical evaluation. The microtiter wells (GMBH+CO KG, Wertheim, Germany) were made of polystyrene (PS). While PS was not used as a tribopair material, we assume that protein adsorption properties of the proteins onto PS would be similar with those onto polyoxymethlylene (POM), which was used as a tribopair material. For the relevance of surface adsorption properties to tribological interfaces of poly(dimethylsiloxane) (PDMS), some microtiter wells were coated with a thin layer of PDMS. To this end, a two-component silicone kit (Sylgard 184, Dow Corning) was employed. Base fluid and crosslinker were mixed at the ratio of 10:1 (w/w). A small drop (ca. $20 \mu \mathrm{L}$ ) was added to each well and cured in an oven at $80^{\circ} \mathrm{C}$ overnight. It is noted that as standard curves were independently obtained for PDM-coated wells too, thin PDMS coating on the absorbance does not affect the adsorbed mass estimation.

\subsection{Pin-on-disk tribometry}

Lubricating properties of the protein solutions at sliding contacts were characterized by pin-on-disk tribometry (CSM, Peseux, Switzerland). In this approach, a loaded spherical pin is allowed to form a contact on a plane disk. The motor-driven rotation of the disk generates interfacial friction forces between the pin and the disk. The applied load is controlled by dead weight and the friction forces 
generated during sliding contact are monitored by a strain gauge. The coefficient of friction, $\mu$, is defined from the relationship, $\mu=F_{\text {friction }} / F_{\text {load. }}$

PDMS was chosen as the tribopair for both pin (6 $\mathrm{mm}$ in diameter) and disk (30 $\mathrm{mm}$ in diameter and $5 \mathrm{~mm}$ in thickness). The pin and disk from PDMS were prepared with the PDMS kit mentioned above as described by Nikogeorgos, Madsen, \& Lee (2014). To ensure that the lubricated contacts were in the boundary lubrication regime, a low sliding speed $(5 \mathrm{~mm} / \mathrm{s})$ was selected under a $5 \mathrm{~N}$ or $2 \mathrm{~N}$ load, corresponding to the apparent maximum Hertzian contact pressure of 0.6 or $0.4 \mathrm{MPa}$, respectively. The friction force data were collected for 100 rotations at room temperature $\left(25^{\circ} \mathrm{C}\right)$ and the tests were repeated multiple times for statistical evaluation. For each measurement, a tribopair of PDMS-PDMS was used only once and discarded to avoid cross contamination between measurements. As a control, a POM pin (6 $\mathrm{mm}$ in diameter) was employed to form a POM/PDMS interface under $5 \mathrm{~N}$ (apparent maximum Hertzian contact pressure of 0.9 $\mathrm{MPa}$ ). The basic mechanical and surface properties of the tribopair materials are presented in Table 1.

\subsection{Mini traction machine (MTM)}

Lubrication properties of the protein solutions were characterized at mixed/rolling contacts in the higher speed regime by means of a mini-traction machine (MTM, PCS Instruments Ltd., UK) too. Mixed rolling/sliding contacts are provided with MTM by independent rotation of ball and disk. The mean speed is defined as [|speed ball - speed $\left._{\text {disk }} \mid / 2\right]$. The slide/roll ratio (SRR) is defined as SRR $=\left(\mid\right.$ speed $_{\text {ball }}-$ speed $\left._{\text {disk }} \mid\right) /\left[\left(\right.\right.$ speed $_{\text {ball }}+$ speed $\left.\left._{\text {disk }}\right) / 2\right] \times 100 \%$, where $0 \%$ SRR represents pure rolling and $200 \%$ SRR represents pure sliding. In this study, SRR of $20 \%$ was employed in all measurements with varying mean speed between $10 \mathrm{~mm} / \mathrm{s}$ to $1200 \mathrm{~mm} / \mathrm{s}$. Tests were conducted at room temperature $\left(25^{\circ} \mathrm{C}\right)$ with the tribopair consisting of a POM ball and a PDMS disk. The PDMS 
disks were prepared from the aforementioned two-component silicone kit (Sylgard 184, Dow Corning) as well. A thick PDMS slab (ca. $5 \mathrm{~mm}$ ) was cast on top of a steel disk (ca. $5 \mathrm{~mm}$ ) for each

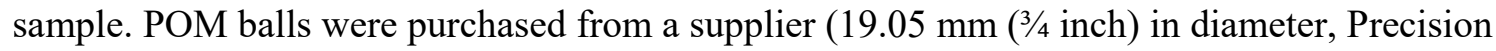
Plastic Ball Co., IL) and were used as received. For each measurement, a new PDMS disk was employed, whereas the same POM ball was used after cleaning with distilled water, ethanol, and sonication in distilled water for 5 min. A fixed load $(2 \mathrm{~N})$ was applied with the estimated Hertzian contact pressure of $0.3 \mathrm{MPa}$. Tests were repeated three times and the friction data were averaged.

\section{Results and discussion}

\subsection{Zeta potentials}

In Figure 1, the zeta potentials of the protein solutions are presented as a function of $\mathrm{pH}$. A zeta potential of near $0 \mathrm{mV}$ was observed at $\mathrm{pH} 5$ for BLG, which is indicative of near-zero net charge and being close to the isoelectric point (IEP) of BLG. As expected, positive and negative zeta potentials were measured when the $\mathrm{pH}$ was shifted to 3 and 7.4, respectively in agreement with the study of Engelhardt et al. (2013). It is noted that despite a gradual decrease in the magnitude of negative charges with decreasing $\mathrm{pH}$, the zero zeta potentials of the mucins were not reached even at $\mathrm{pH} 3$, indicating that the IEPs of both mucins are lower than $\mathrm{pH} 3$ (Lee, Müller, Rezwan, \& Spencer, 2005; Sotres, Madsen, Arnebrant, \& Lee, 2014). It is further to note that BSM displayed more negative zeta potentials than PGM at all $\mathrm{pH}$ values due to higher abundance of negatively charged moieties in BSM, such as sialic acids (bound sialic acid $9-17 \%$ for BSM Type I-S and 0.5 $-1.5 \%$ for PGM Type III, Sigma Aldrich).

For the BLG-BSM mixture, the trend of the zeta potential changes according to $\mathrm{pH}$ change appears to be similar to that of BSM itself, except for slightly less negative values. However, as the absolute values of the zeta potential of BLG at pH 3 and 5 are much smaller than those of BSM at 
these $\mathrm{pHs}$, and as the zeta potentials of BLG and BSM are very similar to each other at $\mathrm{pH} 7.4$, the interaction nature between BSM and BLG cannot be judged based on zeta potential data alone. The seemingly ignorable contribution of BLG to the zeta potential of BLG-PGM mixture at $\mathrm{pH} 5$ could be also discussed in the same context. At pH 3 and 7.4, however, despite fairly different zeta potentials of BLG and PGM, their mixture showed nearly the same zeta potentials of PGM rather than intermediate ones, which signifies the dominance of the electrophoretic mobility of PGM in the mixed protein solutions.

\subsection{Surface adsorption properties}

The adsorbed masses of the proteins per unit area, denoted as $\Gamma$, of the PDMS-coated microtiter well surfaces are presented in Figure 2. It is noted that Figure 2(a) is for the data obtained from 1 $\mathrm{mg} / \mathrm{mL}$ (all protein solutions) and Figure 2(b) is for those from $10 \mathrm{mg} / \mathrm{mL}$ (PGM, BLG, and BLGPGM mixture solutions only). The results for the PS microtiter wells were very similar and the results are shown in Supplementary Information (Figure S3). A few noticeable features of the adsorption behavior of mucins, BLG, and their mixtures are discussed as follows.

Firstly, the adsorbed masses of mucins were clearly higher than those of BLG under the same conditions (concentration and $\mathrm{pH}$ ). For instance, the $\Gamma_{\mathrm{PGM}}$ was ca. $3 \mathrm{mg} / \mathrm{m}^{2}$ and $\Gamma_{\mathrm{BSM}}$ ranged ca. 2.0$4.5 \mathrm{mg} / \mathrm{m}^{2}$ from $1 \mathrm{mg} / \mathrm{mL}$ solutions for all $\mathrm{pH}$ values, whereas $\Gamma_{\text {BLG }}$ ranged ca. $0.4-1.0 \mathrm{mg} / \mathrm{m}^{2}$ only (Figure 3). While the experimental approach employed in this study was markedly different from more conventional optical approaches, the $\Gamma$ values for mucins (Shi, \& Caldwell, 2000; Lee, Müller, Rezwan, \& Spencer, 2005; Nikogeorgos, Madsen, \& Lee, 2014) and BLG (Krisdhasima, McGuire, \& Sproull, 1992) onto hydrophobic surfaces are roughly in the same range, which supports the validity of BCA as a quantitative surface adsorption characterization technique. Given the adsorbed mass per unit area and the molecular weight of BSM (1.6 MDa, Shi, \& Caldwell, 
2000), PGM (1.25 MDa, Davies, \& Viney, 2002), and BLG (18 kDa, Zúñiga, Tolkach, Kulozik, \& Aguilera, 2010), the number of protein molecules per unit area, or conversely, the surface area occupied per single molecule could be estimated. If we further assume that this area is circular, its diameter, $D_{\mathrm{s}}$, can be compared with the hydrodynamic diameter, $D_{\mathrm{h}}$, of the proteins in a bulk solution from literature (Durrer, Irache, Duchene, \& Ponchel, 1995; Celebioglu et al., 2015). The results (Table 2) showed that the $D_{\mathrm{s}, \mathrm{BLG}}$, is comparable to $D_{\mathrm{h}}$, BLG or smaller, whereas the $D_{\mathrm{s}}$, BSM or

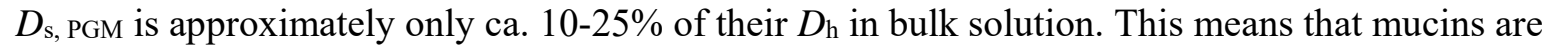
not only higher than BLG in the adsorbed masses, but also adsorb onto the surfaces in a more compact conformation due to a high flexibility of mucins to accommodate themselves in a narrow space or a possibility to form multilayers.

Secondly, $\mathrm{pH}$ was observed to have an influence on the surface adsorption of both mucins and BLG. For instance, BSM showed higher adsorbed masses at acidic $\mathrm{pHs}$ than $\mathrm{pH} 7.4$, in consistent with a recent study by Sotres, Madsen, Arnebrant, \& Lee (2014). This behavior is readily expected from polyanionic characteristics of BSM and nonpolar characteristics of PDMS surface; adsorption of polyanionic species onto nonpolar surfaces from aqueous environment inevitably leads to the accumulation of charges on the surface and it act as a barrier to hamper further adsorption (Sotres, Madsen, Arnebrant, \& Lee, 2014). With decreasing pH, BSM starts to be protonated and the barrier can be diminished. The $\mathrm{pH}$ dependence of PGM for the adsorption onto PDMS at $1 \mathrm{mg} / \mathrm{mL}$ is much weaker, and this is consistent with the fact that PGM carries less charged moieties as shown by the zeta potential measurements (Figure 1). However, at $10 \mathrm{mg} / \mathrm{mL}$, PGM also showed a highest adsorbed mass at pH 3 than at lower pHs, presumably due to the activation of the electrostatic repulsion mechanism mentioned above. Adsorption of BLG onto PDMS surfaces showed a higher $\Gamma_{\mathrm{BLG}}$ values at $\mathrm{pH} 5$ compared to $\mathrm{pH} 3$ and 7.4, which could also be explained by the electrostatic repulsion model, as the zeta potential of BLG is nearly zero at $\mathrm{pH} 5$ (Figure 1). 
Thirdly, and most importantly, mixed protein solutions showed much lower $\Gamma$ values than those of respective mucins and comparable to BLG at each condition. While only a half of the mucin concentration in the mixed protein solutions can be a first reason, this behavior could be ascribed also to the Vroman effect too (Vroman \& Adams, 1969);Lassen \& Malmsten, 1997; Latour, 2008); as BLG is much smaller and lighter than the mucins, it is more mobile and can reach the surface faster than the mucins in the early stage of adsorption ( $\leq 1 \mathrm{hr}$ in this study). Furthermore, as the weight/volume concentration of BLG and mucins were equal in the mixed protein solutions, the number of BLG molecules overwhelms that of mucins due to much smaller molecular weight of BLG. Thus, BLG can readily dominate the initial surface adsorption..

More importantly, the dominance of BLG in the surface adsorption from the BLG-mucin mixture mentioned above implies that there is a large portion of "free" BLG molecules in the mixed protein solutions, and that they participate in the surface adsorption process in competition with the mucins. This is contradicting with a recent spectroscopic study on the interaction between BLG and BSM (Celebioglu et al., 2015), in which the DLS measurements of the BLG-BSM mixture led to a complete disappearance of the peak corresponding to free BLG molecules. This may be caused by the substantially different light scattering sensitivity for the two types of molecules, i.e. BLG molecules are not readily detectable when they are present together with much larger BSM molecules in a solution. It may also simply reflect a very weak interaction nature between BLG and BSM even if they may form loose aggregates.

\subsection{Lubricating properties}

In this study, two types of hydrophobic interfaces, namely PDMS-PDMS and POM-PDMS, were employed for the lubrication studies. Hydrophobic substrates were chosen as they may potentially mimic an oral mucosa membrane underneath mucus layers and facile adsorption of mucins onto the 
substrate can complete an in-vitro oral mucosa model. More importantly, in order to assess the boundary lubricating properties of the proteins, the substrates should effectively attract them onto the surface in the first place, and thus hydrophobicity is a first demanded attribute as the tribopair. POM and PDMS are though very different in their mechanical properties and surface roughness (Table 2). Thus, without substantial changes in the tribological parameters, e.g. external load, these two sliders provide significantly different contact pressure regimes on the opposing substrate.

\subsubsection{Sliding contacts of soft-soft and smooth interface; pin-on-disk tribometry}

Figure 3 shows the $\mu$ values obtained from the sliding contacts of PDMS-PDMS interface as lubricated by the protein solutions at (a) $1 \mathrm{mg} / \mathrm{mL}$ (all protein solutions) and (b) $10 \mathrm{mg} / \mathrm{mL}$ (PGM, BLG, and BLG-PGM mixture solutions only) as characterized by pin-on-disk tribometry. Buffers with three different $\mathrm{pHs}$ showed $\mu$ values of $0.5-0.6$ (Figure $3(\mathrm{a})$ ). At $1 \mathrm{mg} / \mathrm{mL}$ concentration, the BSM solutions displayed exceedingly superior lubricity. This is consistent with previous studies showing effective boundary lubrication by BSM at a PDMS-PDMS interface, yet under much lower load (Nikogeorgos, Madsen, \& Lee, 2014). BSM showed higher $\mu$ values at $\mathrm{pH} 3(\mu \approx 0.2)$ compared to $\mathrm{pH} 5$ and $7.4(\mu \approx 0.02)$, for which the origin is not clearly understood yet. As the $\Gamma_{\mathrm{BSM}}$ values at $\mathrm{pH} 3$ was comparable to those at $\mathrm{pH} 5$ and higher than at $\mathrm{pH} 7.4$ (Figure 2), this behavior cannot be understood in view of surface coverage with BSM at varying $\mathrm{pH}$. One possibility is that charged BSM at pH 7 may lubricate more effectively due to charge-charge repulsion between the opposing surfaces.

PGM solutions showed virtually ignorable lubricating effect, despite comparable adsorbed masses with BSM at the same concentration, $1 \mathrm{mg} / \mathrm{mL}$ (Figure 2). While the lubricity of the PGM solution improved at $\mathrm{pH} 3$, in consistent with a former study (Lee, Müller, Rezwan, \& Spencer, 2005), the $\mu$ values were still somewhat higher than those of BSM at the same condition. It is also 
noticeable that the lubricity of the PGM solutions at $10 \mathrm{mg} / \mathrm{mL}$ is not improving or even inferior to that of PGM at $1 \mathrm{mg} / \mathrm{mL}$ at $\mathrm{pH} 3$ (Figure 3(b)). As a control, an experiment employing the 10 $\mathrm{mg} / \mathrm{mL}$ PGM solution at $\mathrm{pH} 3$ again showed low $\mu$ values under a reduced load of $2 \mathrm{~N}$ (Figure 3(b)), the exceptionally high $\mu$ values of $10 \mathrm{mg} / \mathrm{mL}$ PGM solutions at $\mathrm{pH} 3$ under $5 \mathrm{~N}$ appear to be related to pressure-induced phenomena, such as bridging of PGM molecules between the two opposing PDMS surfaces and consequently high adhesive contacts.

The lubricating efficacy of BLG solutions was also observed to be poor. Although some variation in $\mu$ values according to the $\mathrm{pH}$ change was observed, at both $1 \mathrm{mg} / \mathrm{mL}$ and $10 \mathrm{mg} / \mathrm{mL}$ concentrations, the absolute $\mu$ values were relatively high $(\mu>0.2)$, indicating that this dependence is of little importance. Insignificant lubricity of BLG is firstly resulting from the lack of distinct amphiphilicity, a structural feature required to stabilize the macromolecules on hydrophobic substrates in aqueous environment. Relatively lower adsorbed masses and larger intermolecular distances between BLG on PDMS surface (Table 2) suggest that a strong adhesion between PDMS surfaces may be still active when PDMS-PDMS sliding contact is lubricated with BLG solutions.

\subsubsection{Sliding contacts of hard-soft and rough interface; pin-on-disk tribometry}

Figure 4 shows the $\mu$ values obtained from POM-PDMS interface as lubricated by BSM solution at $\mathrm{pH} 7.4$ or buffer solution under otherwise the same conditions with Figure 3. For a direct comparison, the $\mu$ values from the PDMS-PDMS counterpart (Figure 3) are also presented. About two times higher $\mu$ values observed from the POM-PDMS than the PDMS-PDMS interface in the buffer solutions is ascribed to the substantially higher surface roughness of the POM surface and consequently high local contact pressures (Table 1). Nevertheless, BSM solution displayed consistently more effective lubrication than BLG or the BLG-BSM mixture solutions. The 
332

ineffective lubricity of the PGM solutions for the POM-PDMS interface was also consistent (data not shown).

\subsubsection{Mixed rolling-sliding contacts of hard-soft and rough interface; MTM}

Figure 5 shows the $\mu$ vs. speed plots obtained from the POM-PDMS tribopair lubricated with the protein solutions at $1 \mathrm{mg} / \mathrm{mL}$ (a) at $\mathrm{pH} \mathrm{3,} \mathrm{(b)} \mathrm{pH}$ 5, and (c) $\mathrm{pH} \mathrm{7.4,} \mathrm{as} \mathrm{characterized} \mathrm{with} \mathrm{MTM.} \mathrm{The}$ results showed that all the protein solutions lowered the $\mu$ values compared to the respective buffer solutions (some missing $\mu$ data points in the low-speed regime for the buffer solutions $>1$ ), even including PGM or BLG solutions, which were less lubricious in tribometer experiments. This is probably due to more favorable conditions for lubrication, including higher speed, higher rolling characteristics, and lower apparent contact pressure $(0.3 \mathrm{MPa})$ for the MTM experiments. With increasing mean speed, the $\mu$ values of all the samples started to decrease, reaching as low as 0.03 for the case of BSM at pH 7.4. However, even in the highest speed regime, no characteristic up-turn of $\mu$ values for elastohydrodynamic lubrication (EHL) (de Vincente, Stokes, \& Spikes, 2005; Nalam, Clasohm, Mashaghi, \& Spencer, 2010) was observed. Thus, the dominant lubrication mechanism is thought to be boundary and/or mixed lubrication without separation of the two opposing surfaces by the fluids. Inability to activate EHL mechanism for this contact is largely related to high surface roughness of the POM ball (Table 1), which was also attributed to as a main reason for higher friction forces for this pair in the pin-on-disk tribometry experiments (Figure 4). The root-mean-square roughness $\left(\mathrm{R}_{\mathrm{q}}\right)$ of the POM ball and PDMS disk is $659 \pm 179 \mathrm{~nm}$ and $1.6 \pm$ $0.3 \mathrm{~nm}$, respectively. Therefore, the composite surface roughness of the POM-PDMS interface,

$R_{q, c}=\sqrt{R_{q, P O M}{ }^{2}+R_{q, P D M S}{ }^{2}}$, is nearly identical with that of POM. Thus, for the activation of the 
EHL mechanism, the lubricating film thickness should be at least 3 times larger than $R_{q, c}$ (Røn, \& Lee 2014), i.e. ca. $2 \mu \mathrm{m}$, which is not realistic, especially for aqueous lubrication.

Superior lubricity by the BSM solution to the other protein solutions, in particular at $\mathrm{pH} 7.4$, was observed in consistent with the pin-on-disk tribometry results (Figure 3 and 4). Due to the degraded lubricity of BSM at $\mathrm{pH} 3$, however, the relative difference in $\mu$ values between the protein solutions became blurred, which also was consistent with the pin-on-disk tribometry data (Figure 3). In fact, the $\mu$ values for the PGM solutions were slightly lower than those of BSM at $\mathrm{pH} 3$, but this difference became much smaller in the high-speed regime. The data obtained from $10 \mathrm{mg} / \mathrm{mL}$ solutions of PGM or BLG-PGM (Supplementary Information, Figure S2) were nearly indistinguishable from those obtained from $1 \mathrm{mg} / \mathrm{mL}$ in Figure 5.

\subsubsection{Surface adsorption properties and lubricity; BSM vs PGM}

A strong contrast in the lubricity between BSM and PGM at PDMS-PDMS sliding interface remains elusive to be understood; both mucins are known to be large in molecular weight and comparable to each other (Sandberg, Blom, \& Caldwell, 2009). Both are heavily glycosylated in the central region to similar extents, and are proposed to be adsorbing onto hydrophobic surfaces from water via hydrophobic interaction with unglycosylated $\mathrm{C}$ - and $\mathrm{N}$-terminal regions. The adsorbed masses onto PDMS surface were also fairly comparable for BSM and PGM as shown from the same concentration, $1 \mathrm{mg} / \mathrm{mL}$, in this study (Figure 2).

It should be noted that the aqueous lubrication by adsorption of amphiphilic macromolecules onto hydrophobic surfaces is achieved essentially by hydration of the hydrophobic surfaces and removal of hydrophobic adhesion between the two opposing surfaces (Lee, \& Spencer, 2005). For mucins, this is achieved by respective role of unglycosylated terminal regions acting as an anchor onto the surface and the glycosylated central region acting to recruit water into the interface as 
mentioned above. Critically important for effective lubrication is to sustain the lubricating layer, i.e. mucin film, under persistently applied tribostress, not just to adsorb in high amount under initial tribostress-free condition. Thus, the adsorbed mass determined in the absence of tribostress (Figure 2) provides only a first indication for lubricity. Another related point to note is that as the adsorption of mucins onto hydrophobic surfaces is achieved mainly via hydrophobic interaction, its binding strength is not strong enough to withstand the tribostress as a monolayer coating. For example, a recent study demonstrated that a monolayer coating of BSM on PDMS surface immersed in buffer solution, i.e. without excess BSM in solution, showed an immediate loss of lubricity upon sliding against a PDMS slider and a gradual increase of $\mu$ with increasing rotation on a sliding track (Nikogeorgos, Madsen, \& Lee, 2014). Thus, effective lubrication by a BSM solution at PDMSPDMS or POM-PDMS interfaces is enabled by continuous re-establishment of the lubricating film under tribostress involving the cycles of adsorption-desorption-readsorption of BSM molecules. Thus, the superior lubricity of BSM to PGM should be related to many other factors than the adsorbed mass itself, such as BSM's superior binding strength onto the surface, more optimized conformation to hydrate the surface, as well as faster convection to the surface to re-form the lubricating films, or the combination thereof.

In order to visualize the relationship between the lubrication efficacy and the surface adsorption properties of the proteins in this study, their adsorbed masses, $\Gamma$, are plotted against $\mu$ values obtained from the pin-on-disk tribometry in Figure 6. Because of somewhat extreme behavior of PGM and its mixture with BLG at $10 \mathrm{mg} / \mathrm{mL}$, only the data obtained from $1 \mathrm{mg} / \mathrm{mL}$ protein solutions are displayed. In Figure 6, as the BSM data set lies in the "right-bottom" quadrant, it reflects the case where high adsorbed mass is directly correlated with effective lubrication. The $\mu$ vs. $\Gamma$ plots also display that the relatively higher friction of BSM at $\mathrm{pH} 3$ is not due to the reduced adsorbed mass at that $\mathrm{pH}$ condition. PGM, being placed in the "right-upper" quadrant, clearly 
demonstrates the case where high adsorbed mass is not sufficient for effective boundary lubrication. Lastly, the location of BLG in the "upper-left" quadrant in the plot suggests that the poor adsorption onto PDMS surface is probably the primary reason for its poor boundary lubrication properties.

\subsubsection{Lubricating properties of BLG-mucin mixtures}

Distinctively different lubricating properties of BSM and BLG make it most interesting to explore the effect of mixing the two proteins. As mentioned earlier, this is interesting largely because a previous study by Vardhanabhuti et al. (2011) reported that the addition of a BLG solution to the PDMS-PDMS interface, lubricated by human saliva film, led to a rapid loss of lubricity at an acidic $\mathrm{pH}(3.5)$, but at a much slower pace at $\mathrm{pH} 7$ or 5 .

The relationship in the change of the adsorbed mass and lubricity upon mixing BLG and BSM could be clearly manifested in the $\mu$ vs. $\Gamma$ plots in Figure 6 . Basically, as a group, the BLG-BSM data set is shifted leftwards, yet without shifting upwards with respect to the BSM data set, suggesting that the lubricity of BSM is generally maintained despite significantly reduced adsorbed masses upon mixing with BLG. In more detail, at $\mathrm{pH} 7.4$, a substantial reduction in the adsorbed mass is accompanied with only a slight degradation of BSM's lubricity upon mixing with BLG. If the reduced surface adsorption is related to the competitive adsorption of BLG, this observation is surprising because, under persistently applied tribostress, molecules that adsorb quicker, i.e. BLG, should dominate the tribological interface. A fairly well sustained lubricity of the BLG-BSM mixture compared to the neat BSM solution suggests that BSM rather dominates the tribological properties of the mixture at this $\mathrm{pH}$. One possible explanation is that as the adsorption of BLG onto PDMS surface tends to leave the ample PDMS surface uncovered (see the section 3.2), BSM can readily overlay onto the surface that is pre-occupied with BLG at pH 7.4 and still effectively lubricate the tribological interface. At $\mathrm{pH}$ 5, however, a drastic reduction in both the adsorbed mass 
and lubricity of BLG-BSM mixture compared BSM is observed. This is related to more facile adsorption of BLG onto PDMS surface at $\mathrm{pH} 5$ than at $\mathrm{pH} 3$ or 7.4; the adsorbed mass of BLG at $\mathrm{pH} 5$ was roughly twice those at $\mathrm{pH} 3$ and 7.4 (Figure 2). In turn, this can be attributed to the electrostatic neutrality of BLG at this $\mathrm{pH}$ (Figure 1) and the absence of electrostatic repulsion between BLG molecules in the surface adsorption process. Thus, the dominance of BLG at the tribological interface, i.e. clearly degraded lubricity of BLG-BSM mixture compared to BSM, can be intensified at $\mathrm{pH}$ 5. At $\mathrm{pH} 3$, a reduction in the adsorbed mass without degrading lubricity is observed upon mixing BSM with BLG, similarly with $\mathrm{pH}$ 7.4. However, as the $\mu$ values of BLG and BSM are similar to each other, the dominance of BLG at the tribological interface can be suggested only based on the significantly reduced adsorbed mass.

Overall, a strong $\mathrm{pH}$ dependence of the lubricating properties of BLG-BSM at the PDMSPDMS interface, which can be related to the reported $\mathrm{pH}$ dependence of the lubricating properties of BLG-saliva interaction, was confirmed even on a molecular level interaction. However, more detailed trends are very different in the interaction of BLG-saliva vs. BLG-BSM. Firstly, for the former, strong interaction of BLG with saliva (Vingerhoeds, Blijdenstein, Zoet, \& van Aken, 2005) or mucosa (Withers, Cook, Methven, Gosney, \& Khutoryanskiy, 2013) has well been established and it formulates the ground for the rapid depletion of saliva films from the tribological interface at $\mathrm{pH}$ 3.5. Meanwhile, for the mixed BLG-BSM solution at $1 \mathrm{mg} / \mathrm{mL}$ concentration, competitive adsorption between them onto the tribological interface and its dependence on $\mathrm{pH}$ appears mainly responsible for varying lubricity according to $\mathrm{pH}$ change. This means, however, that BLG and mucins do not formulate tightly bound aggregates in the mixture solution. Secondly, while rapid degradation of lubricity was observed only at $\mathrm{pH} 3.5$ for the BLG-saliva interaction, the degraded lubricity was most prominent at $\mathrm{pH} 5$ for the BLG-BSM interaction, yet much weaker at $\mathrm{pH} 7.4$ or $\mathrm{pH}$ 3. Again, this is due to that the main cause for the degrading lubricity of BLG-BSM mixed 
solution is competitive adsorption onto the tribological interface rather than strong aggregation between them.

For the mixtures of BLG-PGM, as the lubricity of BLG or PGM, as well as the mixed solution of BLG-PGM, is equally poor, the tribology data alone do not provide conclusive information on the interaction between BLG and PGM. Meanwhile, Figure 6 shows that the net effect of mixing BLG and PGM is featured with substantially decreased adsorbed masses compared to the neat PGM solutions. Thus, it can be also suggested that BLG dominates the tribological interface for BLG-PGM mixture.

\section{Conclusions}

In this study, we have investigated the molecular-level interaction between mucins and BLG by means of tribological approaches according to mucin type, solution $\mathrm{pH}$, and protein concentration. Hydrophobic interfaces, namely PDMS-PDMS and POM-PDMS, were employed for feasible adsorption of the proteins and consequent possibility of assessment of the boundary lubricating properties. Surface adsorption properties of the proteins by BCA assay revealed that both mucins adsorbed onto the hydrophobic substrates in a large amount to form either highly compact layers or multilayers, whereas BLG appeared to adsorb without interfering with neighboring molecules or even by partly exposing bare substrates. This difference was firstly related to generally more effective lubricating properties of mucins, in particular BSM, compared to BLG. Nevertheless, nearly ignorable lubricating effect by PGM, despite its facile surface adsorption, suggests that other parameters than adsorbed masses play a significant role to impart superior lubricity of BSM to PGM or BLG. While both pin-on-disk tribometry and MTM were employed to provide the tribological contacts with different contact pressure, speed range, and slide/roll ratio, the dominating lubrication mechanism by the protein solution was boundary lubrication. Surface 
adsorption and lubricating properties of mixed protein solutions, such as BLG-BSM and BLGPGM, with respect to neat protein solutions were of prime interest as it can be compared with the well-known role of BLG as astringency to form a complex with saliva and rapidly deplete from the tribological interface at acidic $\mathrm{pH}$ (3.5, for example). Even in the absence of tribostress, the adsorbed masses of the mixed protein solutions reduced significantly, and BLG appeared to dominate the surface adsorption event, presumably due to the reduced concentration of mucins as well as the Vroman effect. Nevertheless, excellent lubricity was still observed at pH 7.4 and BSM apparently dominated the tribological interface, which highlights the excellent lubricating capabilities of BSM. Although being still relatively more lubricious than the other proteins, the BLG-BSM mixture showed the highest level of degradation in the lubricity of BSM at $\mathrm{pH} 5$, which contrasts the case of BLG-saliva interaction. This is due to that instead of strong aggregation, as in BLG-saliva, the lubricating properties of BLG-BSM are determined by competitive adsorption of the two proteins onto substrates. Most importantly, these observations further suggest that BLG and BSM molecules do not form strong aggregates, especially under tribological stress. PGM's intrinsically weaker lubricity remained largely unchanged even in the interaction with BLG.

\section{Acknowledgements}

The authors would like to thank to the Turkish Government for a PhD scholarship, European Research Council (Funding scheme: ERC Starting Grant 2010, project 261152), and the Danish Strategic Research Council (DSF -10-93456, FENAMI Project) for financial support.

\section{References}

Bansil, R., Stanley, E., \& LaMont, J. T. (1995). Mucin biophysics. Annual Review Physiology, 57, 635-657. 
Berg, I. C. H., Lindh, L., \& Arnebrant, T. (2004). Intraoral lubricaiton of PRP-1, statherin, and mucin as studied by AFM. Biofouling, 20, 65-70.

Celebioglu, H. Y., Guðjónsdóttir, M., Meier, S., Duus, J. Ø., Lee, S., \& Chronakis, I. S. (2015). Spectroscopic studies of the interactions between beta-lactoglobulin and bovine submaxillary mucin. Food Hydrocolloids, 50, 203-210.

Chen, J., Liu, Z., \& Prakash, S. (2014). Lubrication studies of fluid food using a simple experimental set up. Food Hydrocolloids, 42, 100-105.

Chen, J., \& Stokes, J. R. (2012). Rheology and tribology: Two distinctive regimes of food texture sensation. Trends in Food Science \& Technology, 25(1), 4-12.

Chojnicka-Paszun, A., de Jongh, H. H. J., \& de Kruif, C. G. (2012). Sensory perception and lubrication properties of milk: Influence of fat content. International Dairy Journal, 26, 15-22. de Vincente, J., Stokes, J. R., \& Spikes, H. A. (2005). Lubrication properties of non-adsorbing polymer solutions in soft elatohydrodynamic (EHD) contacts. Tribology International, 38, 515-526.

Davies, J. M., \& Viney, C. (1998). Water-mucin phases: conditions for mucus liquid crystallinity. Thermochimica Acta, 315, 39-49.

Durrer, C., Irache, J. M., Duchene, D., \& Ponchel, G. (1995). Mucin interactions with functionalized polystyrene latexes. Journal of Colloid and Interface Science, 170(2), 555-561. Engelhardt, K., Lexis, M., Gochev, G., Konnerth, C., Miller, R., Willenbacher, N., \& Braunschweig, B. (2013). pH Effects on the molecular structure of $\beta$-lactoglobulin modified airwater interfaces and its impact on foam rheology. Langmuir, 29, 11646-11655.

Fang, Y., \& Dalgleish, D. (1997). Conformation of beta-Lactoglobulin studied by FTIR: Effect of $\mathrm{pH}$, temperature, and adsorption to the oil-water Interface. Journal of Colloid and Interface Science, 196, 292-298. 
Joyner Melito, H. S., Pernell, C. W., \& Daubert, C. R. (2014). Impact of formulation and saliva on acid milk gel friction behavior. Journal of Food Science, 79, 67-80.

Krisdhasima, V., McGuire, J., \& Sproull, R. (1992). Surface hydrophobic influences on $\beta$ lactoglobulin adsorption kinetics. Journal of Colloid and Interface Science, 154, 337-350.

Lassen, B., \& Malmsten, M. (1997). Competitive protein adsorption at plasma polymer surfaces. Journal of Colloid and Interface Science, 186, 9-16.

Lee, S., Müller, M., Rezwan, K., \& Spencer, N. D. (2005). Porcine gastric mucin (PGM) at the water/poly(dimethylsiloxane) (PDMS) interface: Influence of $\mathrm{pH}$ and ionic strength on its conformation, adsorption, and aqueous lubrication properties. Langmuir, 21, 8344-8353.

Lee, S., \& Spencer, N. D. (2005). Aqueous lubrication of polymers: Influence of surface modification, Tribology International, 38, 922-930.

Lundin, L., Golding, M., \& Wooster, T. J. (2008). Understanding food structure and function in developing food for appetite control. Nutrition \& Dietetics, 65, S79-S85.

McClements, D. J., Decker, E. A., \& Park, Y. (2009). Controlling lipid bioavailability through physicochemical and structural approaches. Critical Reviews in Food Science and Nutrition, 49(1), $48-67$.

Menchicchi, B., Fuenzalida, J. P., Bobbili, K. B., Hensel, A., Swamy, M. J. Goycoolea, F. M. (2014). Structure of chitosan determines its interactions with mucin. Biomacromolecules, 15, 35503558.

Menchicchi, B., Fuenzalida, J. P., Hensel, A., Swamy, M. J., David, L., Rochas, C., Goycoolea, F. M. (2015). Biophysical analysis of the molecular interactions between polysaccharides and mucin. Biomacromolecules, 16, 924-935.

Meyer, D., Vermulst, J., Tromp, R. H., \& de Hoog, E. H. A. (2011). The Effect of inulin on tribology and sensory profiles of skimmed milk. Journal of Texture Studies, 42, 387-393. 
Nalam, P. C., Clasohm, J. N., Mashaghi, A., \& Spencer, N. D. (2010). Macrotribological studies of poly(L-lysine)-graft-poly(ethylene glycol) in aqueous glycerol mixtures. Tribology Letters, 37, 541552.

Nikogeorgos, N., Madsen, J. B., \& Lee, S. (2014). Influence of impurities and contact scale on the lubricating properties of bovine submaxillary mucin (BSM) films on a hydrophobic surface.

Colloids and Surfaces B: Biointerfaces, 122, 760-6.

Pakkanen, K. I., Madsen, J. B., \& Lee, S. (2015). Conformation of bovine submaxillary mucin layers on hydrophobic surface as studied by biomolecular probes. International Journal of Biological Macromolecules, 72, 790-796.

Prakash, S., Tan, D. D. Y., \& Chen, J. (2013). Applications of tribology in studying food oral processing and texture perception. Food Research International, 54, 1627-1635.

Qaqish, R. B. \& Amiji, M. M. (1999). Synthesis of a fluorescent chitosan dervative and its application for the study of chitosan-mucin interactions. Carbohydrate Polymers, 38, 99-107.

Rossetti, D., Bongaerts, J. H. H., Wantling, E., Stokes, J. R., \& Williamson, A. (2009). Astringency of tea catechins: more than an oral lubrication tactile percept. Food Hydrocolloids, 23(7), 19841992.

Røn, T., \& Lee, S. (2014). Influence of temperature on the frictional properties of water-lubricated surfaces, Lubricants, 177-192.

Sakurai, K., Konuma, T., Yagi, M., \& Goto, Y. (2009). Structural dynamics and folding of betalactoglobulin probed by heteronuclear NMR. Biochimica et Biophysica Acta, 1790(6), 527-37. Sandberg, T., Blom, H., Caldwell, K. D. (2009). Potential use of mucins as biomaterial coatings. I. Fractionation, characterization, and model adsorption of bovine, porcine, and human mucins. Journal of Biomed Materials Research A, 91, 762-772. 
Sandberg, T., Mellina, L., Gelius, U., \& Caldwell, K. D. (2009). Surface analysis of pure and complex mucn coatings on a real-type substrate using individual and combined mBCA, ELLA, and ELISA. Journal of Colloid and Interface Science, 333, 180-187.

Sarkar, A., Goh, K. K. T., \& Singh, H. (2009). Colloidal stability and interactions of milk-proteinstabilized emulsions in an artificial saliva. Food Hydrocolloids, 23, 1270-1278.

Selway, N., \& Stokes, J. R. (2013). Insights into the dynamics of oral lubrication and mouth feel using soft tribology: Differentiating semi-fluid foods with similar rheology. Food Research International, 54, 423-431.

Shi, L., \& Caldwell, K. D., (2000). Mucin adsorption to hdyrophobic surfaces. Journal of colloid and Interface Science, 224, 372-381.

Silletti, E., Vingerhoeds, M. H., Norde, W., \& van Aken, G. A. (2007). The role of electrostatics in saliva-induced emulsion flocculation. Food Hydrocolloids, 21, 596-606.

Singh, H., \& Ye, A. (2013). Structural and biochemical factors affecting the digestion of proteinstabilized emulsions. Current Opinion in Colloid \& Interface Science, 18, 360-370.

Singh, H., Ye, A., \& Horne, D. (2009). Structuring food emulsions in the gastrointestinal tract to modify lipid digestion. Progress in Lipid Research, 48, 92-100.

Smith, P. K., Krohn, R. I., Hermanson, A. K., Mallia, A. K., Gartner, F. H., Provenzano, E. K., Klenk, D. C. (1985). Measurement of Protein Using Bicinchoninic Acid. Analytical Biochemistry, $150,76-85$.

Sotres, J., Madsen, J. B., Arnebrant, T., \& Lee, S. (2014). Adsorption and nanowear properties of bovine submaxillary mucin films on solid surfaces: influence of solution $\mathrm{pH}$ and substrate hydrophobicity. Journal of Colloid and Interface Science, 428, 242-250.

Svensson, O., \& Arnebrant, T. Mucin layers and multilayers - Physicochemical properties and applications. (2010). Current Opinion in Colloid \& Interface Science, 15, 395-405. 
571 Tabak, L. A., Kevine, M. J., Mandel, I., Ellison, S. A. (1982). Role of salivary mucins in the

572 protection of the oral cavity. Journal of Oral Pathology, 11, 1-17.

573 Van Aken, G. A. (2013). Acoustic emission measurement of rubbing and tapping contacts of skin

574 and tongue surfaces in relation to tactile perception. Food Hydrocolloids, 31, 325-331.

575 Van Aken, G. A., Vingerhoeds, M. H., \& de Hoog, E. H. A. (2007). Food colloids under oral

576 conditions. Current Opinion in Colloid \& Interface Science, 12(4-5), 251-262.

577 Vardhanabhuti, B., Cox, P. W., Norton, I. T., \& Foegeding, E. A. (2011). Lubricating properties of

578 human whole saliva as affected by $\beta$-lactoglobulin. Food Hydrocolloids, 25, 1499-1506.

579 Vingerhoeds, M. H., Blijdenstein, T. B. J., Zoet, F. D., \& van Aken, G. A. (2005). Emulsion

580 flocculation induced by saliva and mucin. Food Hydrocolloids, 19, 915-922.

581 Vroman, L., \& Adams, A. L. (1969). Findings with the recording ellipsometer suggesting rapid

582 exchange of specific plasma proteins at liquid/solid interfaces. Surface Science, 16, 438-446.

583 Withers, C. A, Cook, M. T., Methven, L., Gosney, M. a, \& Khutoryanskiy, V. V. (2013).

584 Investigation of milk proteins binding to the oral mucosa. Food \& Function, 4, 1668-1674.

585 Yakubov, G. E., McColl, J., Bongaerts, J. H., \& Ramsden, J. J. (2009). Viscous boundary

586 lubrication of hydrophobic surfaces by mucin. Langmuir, 25, 2313-2321.

587 Zúñiga, R. N., Tolkach, A., Kulozik, U., \& Aguilera, J. M. (2010). Kinetics of formation and

588 physicochemical characterization of thermally-induced beta-lactoglobulin aggregates. Journal of

589 Food Science, 75, 261-268.

590

591 
592 Table 1.

\begin{tabular}{ccccc}
\hline & $\begin{array}{c}\text { Young's modulus } \\
(\mathrm{MPa})\end{array}$ & Poisson ratio & $\begin{array}{c}\text { Surface } \\
\text { roughness } \\
(\mathrm{nm})\end{array}$ & $\begin{array}{c}\text { Static water } \\
\text { contact angle } \\
\left({ }^{\circ}\right)\end{array}$ \\
\hline PDMS & 2 & 0.5 & $1.6 \pm 0.3$ & $105.6 \pm .2$ \\
POM & 3100 & 0.35 & $659 \pm 179$ & $84.8 \pm 2.9$ \\
\hline
\end{tabular}

593

594 
596 Table 2.

\begin{tabular}{|c|c|c|c|c|c|c|c|c|c|c|c|c|}
\hline \multirow{2}{*}{$\begin{array}{l}\text { Samples } \\
\mathrm{pH}\end{array}$} & \multicolumn{3}{|c|}{$\begin{array}{l}\text { Adsorbed mass } \\
\qquad\left(\mathrm{mg} / \mathrm{m}^{2}\right)\end{array}$} & \multicolumn{3}{|c|}{$\begin{array}{c}\text { A, Area per protein } \\
\text { molecule }\left(\mathrm{nm}^{2}\right)\end{array}$} & \multicolumn{3}{|c|}{$\begin{array}{l}D_{\mathrm{s}} \text {, Distance between } \\
\text { protein molecule on } \\
\text { surface }(\mathrm{nm})\end{array}$} & \multicolumn{3}{|c|}{$D_{\mathrm{s}} / D_{\mathrm{h}}$} \\
\hline & 3 & 5 & 7.4 & 3 & 5 & 7.4 & 3 & 5 & 7.4 & 3 & 5 & 7.4 \\
\hline BSM & 2.03 & 4.54 & 2.11 & 1307.6 & 585.2 & 1258.0 & 40.8 & 27.3 & 40.0 & 0.25 & 0.17 & 0.24 \\
\hline PGM & 2.81 & 2.94 & 3.07 & 737.3 & 706.9 & 674.5 & 30.6 & 30.0 & 17.0 & 0.14 & 0.13 & 0.10 \\
\hline BLG & 0.49 & 1.10 & 0.38 & 62.0 & 27.7 & 79.8 & 62.0 & 27.7 & 79.8 & 1.37 & 0.91 & 1.55 \\
\hline
\end{tabular}

597 